\title{
Collisional Dark Matter and the Origin of Massive Black Holes
}

\author{
Jeremiah P. Ostriker
}

\section{Princeton University, Princeton NJ 08544 USA}

The nature of cosmological dark matter remains mysterious. Recently, Spergel and Steinhardt [1] have revived suggestions $[2,3]$ that dark matter may be strongly self-interacting, i.e., collisional, to make galactic halos less dense. We show here an important by-product: for reasonable values of particle mass and collisional cross-section, galaxy cores would quite naturally grow, within them, massive black holes $10^{6}-10^{9} \mathrm{M}_{\odot}$, having the scaling observed by Magorrian et al. [4], $\mathbf{M}_{B H} \propto \mathbf{V}_{c, g a l}^{4.5}$.

The physical picture is quite simple. Assuming a normal power spectrum of perturbations, dark matter halos begin to form in earnest in the redshift range $Z=30 \rightarrow 20$, with star formation commencing in a significant way during the interval $Z=20 \rightarrow 10$ (Ostriker and Gnedin [5], Haiman, Rees and Loeb [6]). The massive stars formed at these early epochs (Abel et al. [7]) will have several dramatic effects on subsequent cosmic evolution: they emit UV radiation copiously, which begins to reheat and reionize the intergalactic medium; they ultimately explode, contaminating their environment with the first heavy elements; and, most importantly for our present purposes, their cores implode to leave black holes (Arnett [8]), having masses $\sim 1 / 4$ of the original stellar mass. The existence of a population III of high mass stars which would have left black hole remnants is attested to by the non-zero $\left(Z / Z_{\odot} \approx 10^{-3}\right)$ floor to the metallicity distribution observed ubiquitously (9) indicating very early and widespread contamination of the universe by ejecta from high mass supernovae. How such stellar mass black holes grow to the supermassive size $\left(10^{6} \lesssim M_{B H} / M_{\odot}<10^{9}\right)$ seen in galactic nuclei [4] remains unknown, although rather general considerations (Rees [10]) indicate the plausibility of their formation. Furthermore, the quantitative scaling observed [4] $M_{B H} \propto M_{g a l} \propto V_{c}^{4.0 \rightarrow 4.5}$ (where $V_{c}$ is the galactic rotation velocity) is an outstanding mystery.

The $25 M_{\odot}$ black hole remnant from a $100 M_{\odot}\left(H, H_{e}\right)$ star will immediately begin to accrete collisional dark matter in the core of the dark halo within which it forms. To frame the discussion, consider the simplest case first. At high densities, the dark matter will behave like an adiabatic gas and accrete as per 
the classic work of Bondi [11]:

$$
\dot{M}_{B H}=\dot{M}_{a c}=4 \pi r_{A}^{2} C_{A} \rho_{A} \text { with } r_{A} \equiv G M_{B H} / C_{A}^{2}
$$

where $\left(\rho_{A}, C_{A}\right)$ are the density and sound speed in the ambient dark matter fluid.

Accretion is treated in the subsequent discussion as if it were quasi-spherical, quite different from the normal treatment of accretion from a rotationally flattened disc. The reason for this is that for normal baryonic matter, electromagnetic radiative losses are usually efficient enough so that energy loss dominates over angular momentum loss in environments of accretion, which leaves the contracting matter in a disc whose further evolution is limited by the rate at which viscosity, feeding from the quasi-Keplerian differential rotation, can transport energy and angular momentum outwards [12]. But in the non-radiative case, both energy and angular momentum can be transported only by collisions (gravitational or physical). Then, as shown by Goodman [13] and others, both transport processes occur on approximately the collisional time scale (i.e., the viscous and conductive time scales are comparable), with angular momentum transported outwards rapidly enough so that the central regions remain quasi-spherical.

Stone et al. [14] have investigated a similar situation of non-radiative accretion in the limit of significant rotation, low viscosity and negligible conduction. They convincingly find both convection and outflow to be important, but it is not likely that their solution applies to the high viscosity, high conductivity, low rotation case considered here. In any case, most of the accretion occurs in the second, optically thin regime which we will address shortly.

We thus return to the simple illustrative example of Bondi - accretion in a quasi-spherical dark matter halo. The core region of which this matter is composed is likely isothermal in its prior structure due to both collisional and violent (Lynden-Bell [15]) relaxation and thus has a profile:

$$
\rho_{A(r)}=C_{A}^{2} /\left(2 \pi G r^{2}\right) .
$$

(We consider a more general profile subsequently.)

Integrating equations (1) and (2), one finds that the central massive black hole grows at the speed of 
sound to reach, in time $t$, a mass $G M_{B H}(t)=2 C_{A}^{3} t$, where we note in passing that it will vacuum up all the local baryonic components as well as the core dark matter.

This phase of rapid growth can only persist until the accretion radius has grown to the point far enough from the center whereby the dark matter mean free path approaches the accretion radius. Then a transition will occur to slower, diffusively limited growth. At this time, $\mathrm{t}_{1}$, mean free path $\lambda=m_{p} /\left(\rho_{t_{1}} \sigma\right)=r_{A}=$ $G M_{B H, t_{1}} / C_{A}^{2}$, or

$$
G M_{B H, t_{1}}=\frac{\sigma_{p}}{2 \pi} \frac{C_{A}^{4}}{G m_{p}}
$$

where $\left(\sigma_{p}, m_{p}\right)$ are the dark matter self-interaction scattering cross-section and particle mass respectively. At this time, the core radius is $\mathrm{r}_{t_{1}}=\sigma_{p} C_{A} /\left(2 \pi G m_{p}\right)$. An alternate way of writing equation (3) in terms of dimensionless numbers is instructive:

$$
\frac{M_{B H, t_{1}}}{m_{e}}=\frac{1}{2}\left(\frac{e^{2}}{G m_{e}^{2}}\right)^{2}\left(\frac{m_{e}}{m_{p}}\right)\left(\frac{\sigma_{p}}{\sigma_{T}}\right)\left(\frac{C_{A}}{c}\right)^{4}
$$

where the first quantity in parenthesis is the classic "big number" representing the ratio of electromagnetic to gravitational forces $\left(\sim 10^{42}\right), \sigma_{T}$ is the electromagnetic Thompson cross-section and $c$ is the speed of light. To translate this into physical units, we take $m_{p}=100 G_{e} V, \sigma / \sigma_{T}=10^{-2}$ and $C_{A}=100 \mathrm{~km} / \mathrm{s}$, then $M_{B H}=3 \times 10^{3} M_{\odot}$, an interesting value.

The next phase of slower growth has been treated by several authors. A cusp forms about the black hole approximately described (for gravitational interactions) by the classic Bahcall-Wolf [16] solution, as modified by loss cone effects (Ostriker and Tremaine [17]) in the inner parts. The rate of accretion is determined by the rate at which particles are scattered into the loss cone, which begins at the Bondi accretion radius. Thus, it is initially the Bondi accretion rate multiplied by the probability of strong scattering for a particle orbiting at the Bondi radius: $P=\sigma C_{A}^{4} /\left(G m_{p} G M_{B H}\right)$, to give an accretion rate $G \dot{M}_{B H}=2 \sigma C_{A}^{7} /\left(G M_{B H}\right)\left(G m_{p}\right)$. This produces a mass growing, after the transition, as the square root of the time, giving for the present time $t_{H} \cdot G M_{B H}=\sqrt{4 \sigma C_{A}^{7} t_{H} /\left(G m_{p}\right)}$, which gives $M_{B H}=4.1 \times 10^{8}$ for the previously quoted parameters, and $t_{H}=10^{10} \mathrm{yrs}$. This produces a somewhat too large value for $M_{B H}$, but one with approximately the 
correct scaling on $C_{A}$. In fact, this solution cannot be extrapolated to late times or to very small values of $\sigma / m_{p}$, because at some point accretion onto the black hole will be limited by the mean free path in the vicinity of the Schwarzschild radius, after which accretion only occurs within a loss cone. Then the estimate is reduced by a factor of $\left(C_{A} / c\right)$ to give (Ostriker and Tremaine [17])

$$
G M_{B H}=\sqrt{I C_{A}^{9} t\left(\sigma / G m_{p}\right) / c^{2}}
$$

where the numerical constant $I$ is approximately $I=\left[\frac{1}{3}+2 \ln \left(c / C_{A}\right)\right] / 2 \pi \approx 2.60$.

Since the fundamental particle physics is quite uncertain in any case, we can best parameterize our ignorance by defining $\eta \equiv \sigma / G m_{p}$ and, noting that the requirement of Spergel and Steinhardt [1] that the halo be optically thick to collisions at a radius $r_{1}$ is equivalent to $1 \equiv 4 r_{1} n_{1} \sigma=2 C_{A}^{2} \sigma / \pi G m_{p} r_{1}$, or $\eta=0.5 \pi r_{1} / C_{A}^{2}$ (it is trivial to generalize these definitions to the likely case that the cross-section is velocity dependent), we find $G M_{B H} \doteqdot 2.0 \sqrt{C_{A}^{7} t_{H} r_{1} / c^{2}}$ or $M_{B H} / M_{\odot}=4.9 \times 10^{7} C_{100}^{7 / 2} t_{H, 10}^{1 / 2} r_{1 k p c}^{1 / 2}$. The range for $r_{1}$ quoted by Spergel and Steinhardt is approximately $0.1 k p c C_{100}^{2}<r_{1}<0.1 M p c C_{100}^{2}$, or $0.45 \mathrm{~cm}^{2} / \mathrm{g}<\sigma / \mathrm{m}_{p}<450 \mathrm{~cm}^{2} / \mathrm{g}$. Then, substitution into equation (5) gives $M_{B H}=7.06 \times 10^{6}\left(\sigma / m_{p}\right)^{1 / 2} V_{c, 100}^{9 / 2} t_{H, 15}^{1 / 2}$ solar masses or, taking the midpoint of the proposed range $\sigma / m_{p}=14.2 \mathrm{~cm}^{2} / \mathrm{g}$, the result $M_{B H}=2.7 \times 10^{7} V_{c, 100}^{9 / 2} t_{H, 15}^{1 / 2} M_{\odot}$, with a range about this $\left(10^{ \pm 0.75}\right)$ encouraged by the Spergel Steinhardt analysis. We replace the speed of sound $C_{A}$ with the circular velocity $V_{c}^{2}=2 C_{A}^{2}$ in the above relation to connect more conveniently to normal astronomical measurements. For both our own galaxy where $V_{c, 100} \approx 2$, and for M87 for which $V_{c, 100} \approx 7$, the resulting black hole masses are too large, as compared to observed estimates [4] by a factor of several hundred. This should be treated as a remarkable agreement given the crudeness both of the analysis and of the estimated collisional DM properties. Accretion in the optically thin limit is only significant for the lower part of this mass range.

Clearly, the assumption of a single stellar mass black hole in the central region of the galaxy is an over simplification, since multiple seeds are expected. Given the instability of the initial growth, one BH is likely to dominate and will eat or eject the others [18]. This complication is not likely to significantly alter the estimates of the final black hole mass. 
It is reasonable to ask how the solution changes if we relax the assumption that the profile is that of a singular isothermal sphere for which the density profile (eq. 2) is $\rho \propto r^{-\alpha}$ with $\alpha=2$. The widely adopted NFW [19] profile for dark matter halos takes $\alpha=1$ in the inner parts, and other authors find typical values near $\alpha=3 / 2$ (see [20,21] for references). One can show [17] that, in the more general case, if the galactic luminosity scales as $L \propto V_{c}^{\mu}$, the mass-to-light ratio scales as $(\mathrm{M} / \mathrm{L}) \propto V_{c}^{\delta}$, so that the mass scales as $M \propto V_{c}^{\mu+\delta}$, then the final black hole mass will scale with the galaxy mass as $M_{B H} \propto M_{\text {gal }}^{K}$, where

$$
K \equiv \frac{9\left[1-\left(1-\frac{\alpha}{2}\right)(\mu+\delta-2)\right]}{\left(\frac{5}{2} \alpha-3\right)(\mu+\delta)}
$$

Remarkably enough, for the case of greatest interest, where $\mu+\delta=9 / 2$, then $\mathrm{K}=1$ regardless of the value of $\alpha$. Thus, the conclusion reached in the case of a cusp which is initially that of a singular isothermal sphere $(\alpha=2)$, that $M_{B H} \propto M_{g a l}$, the observational result found by Magorrian et al. [4], is likely to be very close to that generally obtained for values of the initial cusp parameter departing moderately from this value.

In the most plausible $[20,21]$ range for the parameter $\alpha,(\alpha=1.3 \pm 0.2)$, the core profile would evolve due to the collisional effects, initially reducing the central density $[1,22]$ and lowering the rate of growth of the central BH, i.e., the evolution of the core must be treated simultaneously with the growth of the central BH. Quinlan's [22] numerical work indicates that the collisional evolution of the core ultimately approaches the singular core collapse $(\alpha=2.25)$ solution [23] which would, if it were reached, greatly accelerate the growth of the central black hole. However, for the acceptable range of the parameter $\sigma / m$, this state would not be reached within a Hubble time for all except the most extreme systems (see also Burkert [24]). Also, the rotation of the dark matter halo will not be trivial, but, since the viscous and angular momentum (outward) transport times are the same as the other relevant time scales [13], this is not likely to provide a significant barrier to accretion. Both of these effects reduce the estimated mass of the BH to a level below that given by equation [5]. Nevertheless, the limit on the observed sizes of central galactic black holes (perhaps below the Magorian et. al [4] estimates) probably restricts $\sigma / m_{p}$ to the lower end of the range proposed by Spergel and Steinhardt: $\sigma / m_{p} \lesssim 1 \mathrm{~cm}^{2} / \mathrm{gm}$. However, only detailed numerical calculations (now in progress) will be able to establish a more precise bound. 
It is quite possible that physical processes not included in this elementary treatment could substantially inhibit the growth of black holes in the Spergel-Steinhardt scenario, the required values being somewhat smaller than given by equation [5]. We must conclude that the existence of strongly self-interacting dark matter has the exciting potential for leading to the growth of central massive black holes in normal galaxies with the observed scaling parameters.

Three corollary consequences should be noted. First, since the hypothesized dark matter particles do not have radiative interactions, accretion of them will not produce an electromagnetic luminosity output, $L_{e m}$, breaking the assumed link $\int L_{e m} d t=\epsilon_{e m} \Delta M / c^{2}$, with normal estimates of $\epsilon_{e m} \approx 0.1$. Alternatively phrased, if most of the accreted matter is dark matter, then a low efficiency $\left(\epsilon_{e m}<<1\right)$ is to be expected. Second, since the mean free path of the particles is comparable to the system size, the dark matter fluid will be extremely viscous, with dynamical consequences that may be imagined. Finally, dark matter galactic halos in clusters of galaxies will tend to evaporate due to heat transfer from the hotter, cluster

dark matter. Preliminary estimates of the significance of this effect again limit one to consider the range $\left(\sigma / m_{p}\right) \lesssim 1 \mathrm{~cm}^{2} / \mathrm{gm}$

\section{ACKNOWLEDGMENTS}

I would like to thank Bohdan Paczynski, Martin Rees, David Spergel, Paul Steinhardt, Scott Tremaine, Neil Turok and Simon White for very helpful conversations, the hospitality of the Newton Institute in Cambridge and the Max Planck Institut for Astrophysics in Garching, where the work was begun, and NSF grants AST-9424416, AST-9803137, and ASC-9740300.

\section{REFERENCES}

[1] D.N. Spergel. and P.J. Steinhardt, Phys. Rev. Letters, (accepted) astro-ph/9909386 (1999).

[2\} E.D. Carlson, M.E. Machacek and L.J. Hall, ApJ, 398, 43-52 (1992).

[3] M.E. Machacek, ApJ, 431, 41-51 (1994).

[4] J. Magorrian, S. Tremaine, D. Richstone,R. Bender, G. Bower, A. Dressler, S.M. Faber, K. Gebhardt, R. Green, C. Grillmair, J. Kormendy, and T. Lauer, Astron. J., 115, 2285 (1998).

[5] J.P. Ostriker and N. Gnedin, ApJ Letters, 472, L63-L67 (1996).

[6] Z. Haiman, M. Rees and A. Loeb, ApJ, 476, 458 (1997). 
[7] T.A. Abel, Anninos, M.L. Norman and P., Y. Zhang, ApJ, 508, 518-520 (1998).

[8] D. Arnett, Supernovae and Nucleosynthesis (Princeton University Press, Princeton, 1996).

[9] P. Hodge, An. Rev. Ast. ES Astroph., 27, 139 (1989).

[10] M. Rees, "Black Holes and Relativity", in Astrophysical Evidence for Black Holes, edited by R. Wald (University of Chicago Press, 1998).

[11] H. Bondi, Mon. Not. Royal Astron. Soc., 107, 410 (1947).

[12] S. S. Holt and T.R. Kallman, AIP Conf. Proc. \#431, Accretion processes in Astrophysics Systems (1998).

[13] J. Goodman, Dynamical Relaxation in Stellar Systems, Ph.D. Thesis, Princeton University (1983).

[14] J. Stone, J.E. Pringle, M.C. Begelman MNRAS, (in press) (2000).

[15] D. Lynden-Bell, Mon. Not. Royal Astron. Soc., 136, 101 (1967).

[16] J.N. Bahcall, J. and R.A. Wolf, ApJ, 209, 214, (1976).

[17] J.P. Ostriker and S. Tremaine (in preparation) (1999).

[18[ M.C. Begelman, R.D. Blandford and M.J. Rees, Nature, 287, 309 (1980); G. Xu and J.P. Ostriker, ApJ, 437, 184 (1994).

[19] J.F. Navarro, C.S. Frenk and S.D.M. White, Mon. Not. Royal Astron. Soc., 275, 720 (1995).

[20] K. Subramanian, R. Cen and J.P. Ostriker, ApJ, (accepted) astro-ph/9909279)(1999).

[21] Y.P. Jing and Y. Suto, astro-ph/0001288 (2000).

[22] G. Quinlan, New Astronomy, 1, 255 (1996).

[23] H. Cohen, ApJ, 242, 765 (1980).

[24] A. Burkert, astro-ph/0002409)(2000). 